\title{
Primary extraosseous plasmacytoma of the parotid gland: A case report and literature review
}

\author{
LUIS MIGUEL GONZALEZ-PEREZ ${ }^{1}$, PEDRO INFANTE-COSSIO ${ }^{1}$ and JUAN JOSE BORRERO-MARTIN ${ }^{2}$
}

\author{
Departments of ${ }^{1}$ Oral and Maxillofacial Surgery, and ${ }^{2}$ Pathology, Virgen del Rocio University Hospital, 41013 Seville, Spain
}

Received April 29, 2017; Accepted June 29, 2017

DOI: $10.3892 / \mathrm{mco} .2017 .1417$

\begin{abstract}
Extraosseous plasmacytoma (EOP) is an uncommon malignant tumour that is characterised by the monoclonal proliferation of abnormal plasma cells in soft tissue; however, EOP lacks the defining features of multiple myeloma or medullary plasmacytoma. Although the majority of EOP lesions occur in the head and neck, EOP of the parotid gland is extremely uncommon. The present study aimed to explore the clinical features of parotid plasmacytoma, in addition to the diagnostic and therapeutic options for its management. Using the Medline database, a search was conducted for articles published on the topic of 'parotid plasmacytoma' up until the year 2016. A total of 20 cases were evaluated, including 19 clinical cases from the literature and 1 new clinical case from our hospital. Among the 19 previously published cases, the mean age at the time of diagnosis of EOP was 65.1 \pm 10.9 years (range, 38-78 years). Plasmacytomas were located unilaterally in all cases: On the right side in 9 patients (47.4\%), on the left side in 10 patients (52.6\%). Treatment included chemotherapy in 3 cases, radiotherapy in 11 cases and surgical removal in 15 cases. The diagnosis of EOP is based on the presence of a localised tumour comprising monoclonal plasma cells, and EOP is identical to multiple myeloma in this regard; however, EOP, in contrast to multiple myeloma, does not exhibit the signs that are indicative of disseminated disease, such as additional lesions on skeletal radiological examination, plasmacytosis in the bone marrow, and hypercalcaemia, anaemia, or renal failure. Thus, EOP must be considered in the differential diagnosis of parotid gland lesions in order to avoid confusion with other tumoural diseases.
\end{abstract}

\section{Introduction}

Plasma cell tumours are lymphoid B-cell neoplasms that are composed of plasma cells. These tumours may develop in a

Correspondence to: Dr Luis Miguel Gonzalez-Perez, Department of Oral and Maxillofacial Surgery, Virgen del Rocio University Hospital, Avenida Manuel Siurot s/n, 41013 Seville, Spain

E-mail: lumigon@icloud.com; lumigon@telefonica.net

Key words: extraosseous plasmacytoma, parotid gland tumour, salivary gland, plasma cell tumour disseminated manner, affecting numerous bones (multiple myeloma), or, more rarely, as a solitary lesion in a single bone (solitary bone medullary plasmacytoma) or soft tissue [extramedullary/extraosseous plasmacytoma (EOP)]. EOP is uncommon, accounting for $\sim 5 \%$ of all plasma cell neoplasms, and arises outside of the bone marrow, unaccompanied by any clinical evidence of existing multiple myeloma. The median age at diagnosis is $\sim 55$ years, and approximately two out of three patients are male $(1,2)$. In $\sim 80 \%$ of cases of EOP, the neoplasm arises in the upper respiratory tract, including the oropharynx, nasopharynx, and sinuses; however, EOP may be located at various other sites, such as the lymph nodes, bladder, digestive system, breast, thyroid, central nervous system, and skin (1).

The clinical manifestations and symptoms of EOP, where present, are non-specific, as these depend on the location of the tumour. EOP manifests as a sessile or pediculate outgrowth, which may be either circumscribed or infiltrating $(1,2)$. Following the diagnosis of the tumour locally, it is necessary to exclude the existence of any systemic processes in order to confirm the diagnosis of EOP. Following treatment, $70 \%$ of patients remain in complete remission for at least 10 years. However, in $\sim 25 \%$ of cases, regional recurrences eventually develop, and metastasis to distant extraosseous sites also occurs occasionally (2). The present report provides a literature review of cases of parotid plasmacytoma published up until 2016, in addition to a presentation of one new clinical case taken from the personal experience of the authors.

\section{Case report}

A 47-year-old man was referred to the Maxillofacial Surgery Clinic at Virgen del Rocio University Hospital (Seville, Spain) in January 2015, with a 3-month history of a painless lesion in the retroauricular area that had gradually increased in size. The patient reported a medical history that included arterial hypertension and sacrococcygeal trauma. Physical examination revealed a $3 \times 3-\mathrm{cm}$, lobulated mass in the right parotid area, which was moderately tender upon palpation (Fig. 1). The facial nerve was intact, and there was no evidence of palpable cervical lymph nodes. Clinical examination was otherwise non-contributory.

On ultrasound, a mass of reduced echogenicity was detected, without any evidence of cervical lymph node enlargement. Magnetic resonance imaging of the head and 
Table I. Overview of published cases of parotid extraosseous plasmacytoma.

\begin{tabular}{|c|c|c|c|c|}
\hline Authors, year & Age/sex & Side & Treatment & (Refs.) \\
\hline Vainio-Mattila & $74 / \mathrm{F}$ & Left & $S+R T$ & (3) \\
\hline \multirow[t]{2}{*}{ Pahor } & $61 / F$ & Left & $\mathrm{S}+\mathrm{CT}+\mathrm{RT}$ & (4) \\
\hline & $69 / \mathrm{M}$ & Right & $\mathrm{S}+\mathrm{CT}+\mathrm{RT}$ & \\
\hline Ferlito et al & $47 / M$ & Left & $\mathrm{S}+\mathrm{RT}+\mathrm{CT}$ & (5) \\
\hline Kurihara and Hashimoto & $61 / \mathrm{F}$ & Right & S & (6) \\
\hline Edney et al & $38 / \mathrm{M}$ & Right & $\mathrm{S}$ & (7) \\
\hline Kanoh et al & $78 / \mathrm{F}$ & Left & $\mathrm{S}+\mathrm{RT}$ & (8) \\
\hline Ebbers & 68/M & Right & RT & (9) \\
\hline Scholl and Jafek & $60 / \mathrm{F}$ & Left & $\mathrm{S}$ & (10) \\
\hline Simi et al & $58 / \mathrm{M}$ & Right & $S$ & (11) \\
\hline Rothfield et al & $53 / \mathrm{M}$ & Left & $S$ & (12) \\
\hline Kerr and Dort & $73 / \mathrm{F}$ & Right & $\mathrm{S}+\mathrm{RT}$ & (13) \\
\hline El-Naggar et al & 73/M & Left & $\mathrm{S}$ & (14) \\
\hline Gonzalez-Garcia et al & $63 / \mathrm{M}$ & Right & $\mathrm{S}+\mathrm{RT}$ & (15) \\
\hline Hari and Roblin & $60 / \mathrm{F}$ & Right & $\mathrm{S}$ & (16) \\
\hline Ustun et al & $77 / \mathrm{F}$ & Left & $S+R T$ & (17) \\
\hline Kanthan and Torkian & $73 / \mathrm{F}$ & Right & $\mathrm{S}+\mathrm{RT}+\mathrm{CT}$ & (18) \\
\hline Gouveris et al & 76/M & Left & RT & (19) \\
\hline Alabed et al & $75 / \mathrm{F}$ & Left & RT & (20) \\
\hline Our case & $47 / \mathrm{M}$ & Right & $\mathrm{S}+\mathrm{RT}$ & - \\
\hline
\end{tabular}

S, surgery; RT, radiotherapy; CT, chemotherapy.

neck revealed a right parotid tail mass in the superficial portion of the right parotid gland. The mass measured $3 \times 3 \times 3 \mathrm{~cm}$, was round with well-defined contours, and appeared hypointense on $\mathrm{T} 1$ and $\mathrm{T} 2$ sequences, and hyperintense on $\mathrm{T} 2$-short tau inversion recovery sequences (Fig. 2). Ultrasonographically guided fine-needle aspiration cytology was performed, and the subsequent cytological analysis demonstrated diffuse infiltration of neoplastic large monoclonal plasmacytes with variable pleomorphism. This finding was suggestive of a lymphoproliferative lesion. Under general anaesthesia, a superficial parotidectomy was performed, following which the patient had an uneventful course and was discharged on the third postoperative day, and followed up at the outpatient clinic. Evaluation of the parotid gland was subsequently performed. The histological examination of tissue sections stained with haematoxylin and eosin showing an intraglandular lymph node with a diffuse plasmacytic proliferation among residual reactive lymphoid follicles, with minimal pleomorphism (some large nuclei and binucleated cells) (Fig. 3). An immunohistochemical study revealed monotypic $\lambda$ light chain restriction, as well as nuclear immunostaining for cyclin D1 (Figs. 4 and 5), and aberrant expression of CD10 (positive expression, which is not associated with the specific cell type). CD56 was found to be negative. No monocytic or marginal lymphoid proliferation was observed; therefore, a diagnosis of cyclin D1-positive, $\lambda$ plasmacytic plasmacytoma was determined.

To differentiate between plasmacytoma and myeloma, a systemic workup was performed. A skeletal radiographic survey revealed no evidence of extraoral involvement on

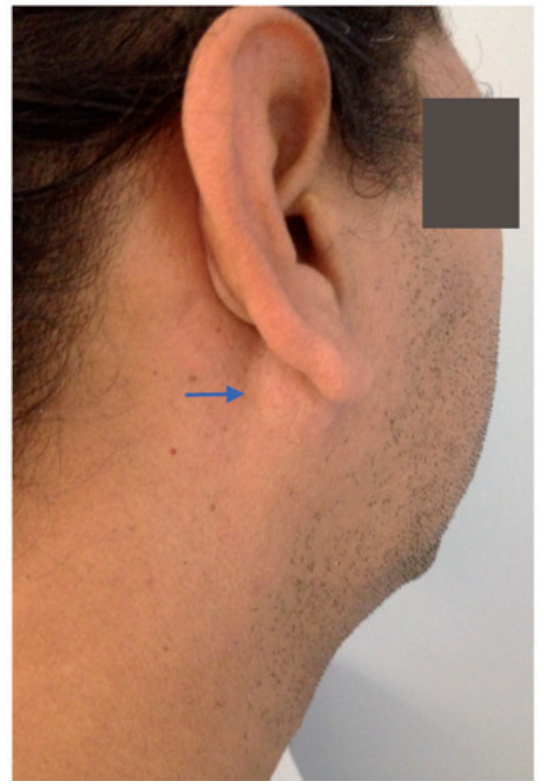

Figure 1. Clinical appearance of the parotid region in the right retroauricular area (arrow) of a patient with a 3-month history of a painless lesion that gradually increased in size.

computed tomography of the chest, abdomen, or pelvis; and positron emission tomography with $18 \mathrm{~F}$-fludeoxyglucose (FDG) showed a hypermetabolic lesion (standardised uptake value, 3.9) with a soft tissue component in the right cervical area, which coincided with the parotid tail mass detected on the ultrasound study. No FDG-avid lymphadenopathy or 


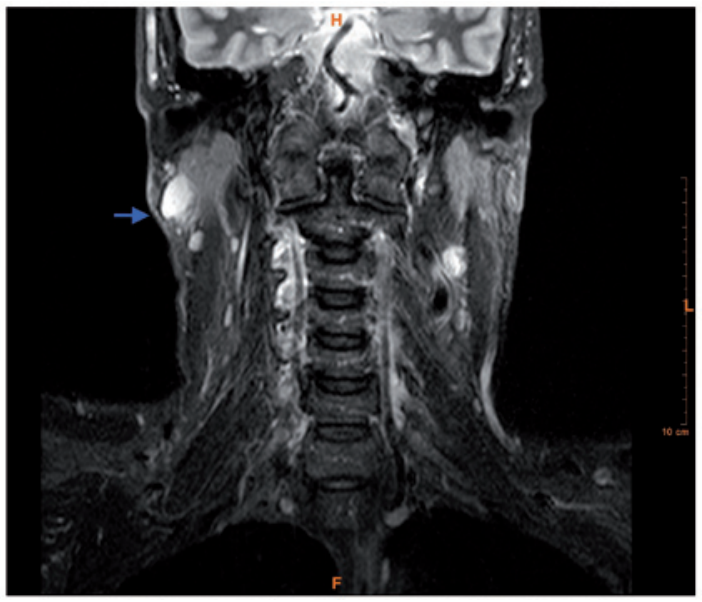

Figure 2. Magnetic resonance imaging of the head and neck demonstrated a right parotid tail mass, measuring $3 \times 3 \times 3 \mathrm{~cm}$. The mass was round, with well-defined contours, and was hypointense on T1 and T2 sequences and hyperintense on the T2-STIR sequence in the superficial portion of the right parotid gland (arrow).

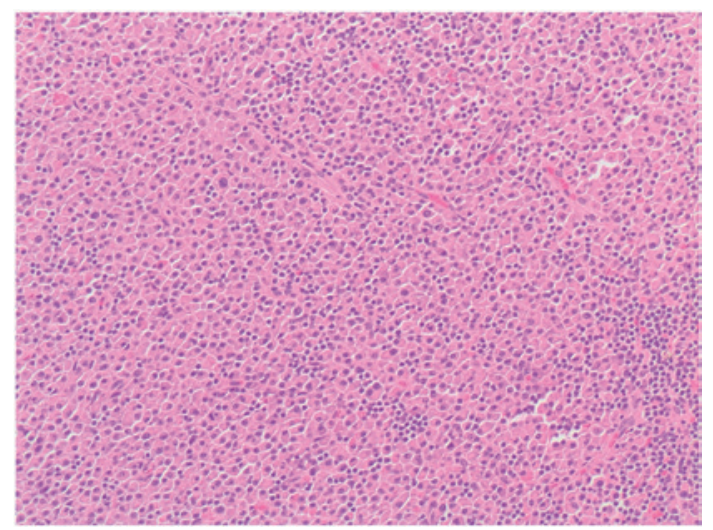

Figure 3. Haematoxylin and eosin staining revealed diffuse plasmacytic proliferation among residual reactive lymphoid follicles, with minimal pleomorphism (a number of large nuclei and binucleated cells). Original magnification, $\mathrm{x} 100$.

distant metastasis was detected. A bone marrow biopsy was negative for malignancy, and laboratory analysis did not reveal anaemia, hypercalcaemia or renal involvement. Assays for Bence-Jones protein in the urine and for serum myeloma protein were negative. A final diagnosis of primary extraosseous/extramedullary plasmacytoma was made. Due to the aggressive nature of the disease, postoperative radiotherapy of the parotid region and neck, including the supraclavicular lymph node area, was performed, with a total dose of $30 \mathrm{~Gy}$. The patient has been followed-up closely for 2 years, during which time he has remained completely devoid of any clinical symptoms, and shows no evidence of recurrence of the parotid tumour.

Written informed consent was obtained from the patient for publication of this case report and accompanying images.

\section{Discussion}

For the present study, a literature search of Medline/PubMed (http://www.ncbi.nlm.nih.gov/pubMed) that included articles

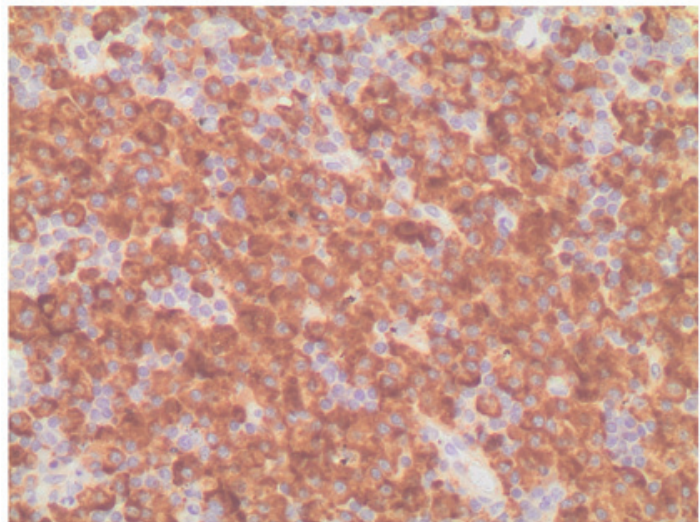

Figure 4. Immunohistochemical staining revealed monotypic $\lambda$ light chain restriction.

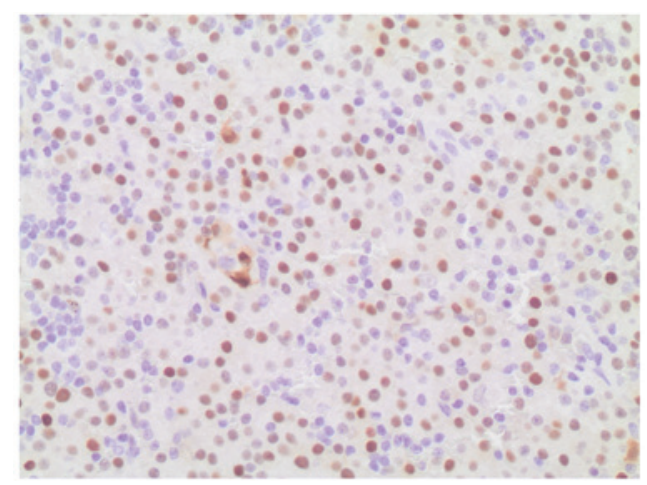

Figure 5. Immunohistochemical staining revealed nuclear expression of cyclin D1.

published up to June 2016 was conducted using the search term 'parotid plasmacytoma'. The inclusion criteria were as follows: English language articles identified in the Medline database, containing at least one case of plasmacytoma located in the parotid gland, along with detailed clinical, diagnostic or therapeutic criteria. The search revealed 18 papers, encompassing a total of 19 cases, that met these criteria. In total, 20 patients with plasmacytoma in the parotid gland were evaluated, including the 19 clinical cases taken from the literature, and the new clinical case presented in the current study (Table I). The mean age of the patients at the time of diagnosis, excluding the present case case, was $65.1 \pm 10.9$ years (range, $38-78$ years). The cases comprised 10 female patients $(52.6 \%)$ and 9 male patients (47.4\%), with parotid plasmacytoma located unilaterally in all cases: 10 patients showed left-side involvement, while 9 cases exhibited right-side involvement. The most frequent signs and symptoms were pain and swelling on the ipsilateral side. Treatment included chemotherapy in 3 cases, radiotherapy in 11 cases, and surgical removal in 15 cases.

A plasmacytoma is a solitary mass of neoplastic monoclonal plasma cells, which can arise in any area of the body. EOP arises from the plasma cells located in areas of soft tissue, whereas solitary bone medullary plasmacytoma arises from plasma cells located in the bone marrow. These two forms of disease represent different groups of neoplasms with regard to location, tumour progression, and overall survival rate $(1,2)$. The diagnosis of EOP, which occurs far less commonly than 
that of solitary plasmacytoma of the bone (1), is determined based on the identification of a localised tumour comprising monoclonal plasma cells, which are identical to those observed in multiple myeloma; however, EOP is distinguished by an absence of indicators of the disseminated form of disease, such as additional lesions detected on skeletal radiological imaging, the presence of bone marrow plasmacytosis, and the presence of anaemia, hypercalcaemia, or renal failure, as was the case for the current patient. Solitary primary EOP is rare, and is predominantly diagnosed in elderly individuals $(4,13,15)$. EOP occurs most often in the head and neck area, and tends to involve the submucosal tissues of the upper respiratory tract, accounting for $\sim 0.5 \%$ of all neoplasms that occur at this location. Involvement of the parotid gland is extremely rare, as evidenced by the fact that only 19 cases (Table I) (3-20) have been previously documented in the published literature, including the first case described in 1965 by Vainio-Mattila (3). Although the current literature review suggested that parotid gland EOP has a favourable prognosis, assuming that multiple myeloma has been ruled out, the clinical behaviour of this disease is not well understood. The conversion rate of solitary EOP to solitary plasmacytoma of the bone is $48 \%$, while that of EOP to multiple myeloma is $20 \%$, both of which are associated with a poorer prognosis $(1,2,20)$. A definitive diagnosis of parotid EOP cannot be established solely by preoperative investigations, as demonstrated in the present case; however, it remains important to recognise this distinctive type of plasmacytoma confined to the parotid area, in order to avoid confusion with other parotid benign lesions/reactive enlargements that have a similar appearance.

As mentioned previously, histopathological analysis in isolation is insufficient to confirm a diagnosis of primary extramedullary plasmacytoma, since it is necessary to exclude the possibility of multiple myeloma through skeletal and marrow examinations, immunoelectrophoresis, quantitative immunoglobulin testing, and confirmation of the absence of urinary Bence Jones proteins (17). Cyclin D1 expression in EOP, as observed in the current patient, has been associated with an unfavourable prognosis in previous studies (19); however, this did not seem to be true of the present case. In addition, the distinction between plasmacytoma and lymphoma with extreme plasma cell differentiation may be challenging $(1,2)$.

The treatment of solitary extramedullary plasmacytoma should consist primarily of eradication of the lesion locally, and surgery therefore appears to be the primary line of treatment. Radiation therapy has also been used in some cases, either alone in patients deemed unsuitable for surgery, or as an adjunct to the surgical removal of the lesion in order to prevent local relapse, as in the current case. The rates of conversion of solitary EOP to solitary plasmacytoma of the bone and to multiple myeloma are 48 and $20 \%$, respectively, and both are associated with a poorer prognosis (1-20). Although not scientifically validated, chemotherapy is theoretically advantageous: As well as augmenting local control of the neoplasm, it may potentially eradicate sub-clinical disease, delaying or preventing the development of myeloma (2). Patterns of primary extramedullary plasmacytoma relapse include local recurrence, transformation to multiple myeloma, and metastasis. Recurrences are typically localized and respond well to radiotherapy. Combined chemotherapy and radiation therapy is recommended for high-risk surgical patients (ASA physical status classification system category $\geq 3$ ), to increase the rates of local control and cure $(2,18-20)$.

In summary, although patients with parotid enlargements are commonly observed in everyday clinical practice, EOP arising in the parotid gland is extremely rare. Fine-needle aspiration cytology, histopathological examination, immunohistochemical analysis, and an absence of signs indicating disseminated disease aid in providing an accurate diagnosis and treatment. EOP should be considered in the differential diagnosis of parotid tumours.

\section{References}

1. McKenna RW, Kyle RA, Kuehl WM, Grogan TM, Harris NL and Coupland RW: Plasma Cell Neoplasms. In: Swerdlow SH, Campo E, Harris NL. (eds). WHO Classification of Tumours of Haematopoietic and Lymphoid Tissues, Lyon, IARC Press, pp200-213, 2008.

2. Bachar G, Goldstein D, Brown D, Tsang R, Lockwood G, Perez-Ordonez B and Irish J: Solitary extramedullary plasmacytoma of the head and neck-long-term outcome analysis of 68 cases. Head Neck 30: 1012-1019, 2008.

3. Vainio-Mattila J: Plasmacytoma of the parotid gland. Arch Otolaryngol 82: 635-637, 1965.

4. Pahor AL: Extramedullary plasmacytoma of the head and neck, parotid and submandibular salivary glands. J Laryngol Otol 91: 241-258, 1977.

5. Ferlito A, Polidoro F and Recher G: Extramedullary plasmacytoma of the parotid gland. Laryngoscope 90: 486-493, 1980.

6. Kurihara K and Hashimoto N: Extramedullary plasmacytoma associated with a Castleman's lesion of the cervical nodes. J Oral Pathol 12: 131-138, 1983.

7. Edney JA, Thompson JS, Conley MC and Moore GE: Plasmacytoma of the parotid gland. J Surg Oncol 28: 165-167, 1985.

8. Kanoh T, Hattori N, Uchino H, Fujita A, Ohmura M and Makimoto K: Extramedullary plasmacytoma of the parotid gland: Report of a case and review of the literature. Tohoku J Exp Med 146: 469-478, 1985.

9. Ebbers J: Plasmacytoma of the parotid gland. Laryngol Rhinol Otol (Stuttg) 65: 127-129, 1986 (In German).

10. Scholl P and Jafek BW: Extramedullary plasmacytoma of the parotid gland. Ear Nose Throat J 65: 564-567, 1986.

11. Simi U, Marchetti G, Bruno R, Di Nasso F and Cardini M: Plasmacytoma of the parotid gland: Report of a case and review of the world literature. Acta Otorhinolaryngol Belg 42: 93-96, 1988.

12. Rothfield RE, Johnson JT and Stavrides A: Extramedullary plasmacytoma of the parotid. Head Neck 12: 352-354, 1990.

13. Kerr PD and Dort JC: Primary extramedullary plasmacytoma of the salivary glands. J Laryngol Otol 105: 687-692, 1991.

14. el-Naggar AK, Ordonez NG and Batsakis JG: Parotid gland plasmacytoma with crystalline deposits. Oral Surg Oral Med Oral Pathol 71: 206-208, 1991.

15. Gonzalez-Garcia J, Ghufoor K, Sandhu G, Thorpe PA and Hadley J: Primary extramedullary plasmacytoma of the parotid gland: A case report and review of the literature. J Laryngol Otol 112: 179-181, 1998

16. Hari CK and Roblin DG: Solitary plasmacytoma of the parotid gland. Int J Clin Pract 54: 197-198, 2000.

17. Ustun MO, Ekinci N and Payzin B: Extramedullary plasmacytoma of the parotid gland. Report of a case with extensive amyloid deposition masking the cytologic and histopathologic picture. Acta Cytol 45: 449-453, 2001.

18. Kanthan R and Torkian B: Solitary plasmacytoma of the parotid gland with crystalline inclusions: A case report. World J Surg Oncol 1: 12, 2003.

19. Gouveris H, Hansen T and Franke K: Solitary extramedullary plasmacytoma and granulomatous sialadenitis of the parotid gland preceding a B-cell non-Hodgkin's lymphoma. Mund Kiefer Gesichtschir 10: 122-125, 2006.

20. Alabed YZ, Rakheja R and Laufer J: Solitary extramedullary plasmacytoma of the parotid gland imaged with 18F-FDG PET/CT. Clin Nucl Med 39: 549-550, 2014. 